\title{
Ultra-thin $\mathrm{ZrO} 2 / \mathrm{SrO} / \mathrm{ZrO} 2$ insulating stacks for future dynamic random access memory capacitor applications
}

Steve Knebel, ${ }^{1}$ Milan Pešić, ${ }^{1}$ Kyuho Cho, ${ }^{2}$ Jaewan Chang, ${ }^{2}$ Hanjin Lim, ${ }^{2}$ Nadiia Kolomiiets, ${ }^{3}$ Valeri V. Afanas'ev, ${ }^{3}$ Uwe Schroeder, ${ }^{1}$ and Thomas Mikolajick ${ }^{1,4}$

\author{
${ }^{1}$ NaMLab gGmbH, Noethnitzer Strasse 64, 01187 Dresden, Germany \\ ${ }^{2}$ Samsung Electronics, 1, Samsungeonja-ro, Hwasung-si, Gyeonggi-do, 445-701, Korea \\ ${ }^{3}$ Katholieke Universiteit Leuven, Leuven, Belgium \\ ${ }^{4}$ Chair of Nanoelectronic Devices, Noethnitzer Strasse 64, Technische Universität Dresden, Dresden, \\ Germany
}

Metal-insulator-metal thin film capacitors with $\mathrm{ZrO}_{2} / \mathrm{Al}_{2} \mathrm{O}_{3} / \mathrm{ZrO}_{2}$ as insulator are common for state of the art $20 \mathrm{~nm}$ DRAM technology applications. For the first time, $\mathrm{ZrO}_{2} / \mathrm{SrO} / \mathrm{ZrO}_{2}$ thin films with TiN electrodes are deposited by physical vapor deposition. Electrical characterization is done and compared with $\mathrm{ZrO}_{2} / \mathrm{Al}_{2} \mathrm{O}_{3} / \mathrm{ZrO}_{2}$ and pure $\mathrm{ZrO}_{2}$ metal-insulator-metal capacitor stacks. Leakage current and capacitance measurement data are presented. Additionally, the metal/insulator interface is investigated by internal photoemission spectroscopy. Band gap and conduction band offset are extracted. Since the dielectric lifetime is one critical issue for modern DRAM, voltage ramp stress was applied on the sample to extract breakdown and stress induced leakage characteristics.

\section{INTRODUCTION}

For many years, the dynamic random access memory (DRAM) was the scaling driver in semiconductor industry. Continuous downscaling of the cell dimension led to the introduction of high-k materials in a 3dimensional cylindrical capacitor geometry with metal electrodes. Currently, the most common DRAM capacitor consists of a symmetrical $\mathrm{ZrO}_{2} / \mathrm{Al}_{2} \mathrm{O}_{3} / \mathrm{ZrO}_{2}$ (ZAZ) stack [1-3]. Intensive research is done on strontium titanate (STO) based capacitors, but here the DRAM target thickness of $<6 \mathrm{~nm}$ is very critical to reach [4-5]. Accordingly, additional research is necessary to optimize the current $\mathrm{ZrO}_{2}$ based material stack.

Thin $\mathrm{ZrO}_{2}$ films crystallize in the tetragonal phase and have a permittivity $\mathrm{k}$ of around 40 but suffer from high leakage current [6]. Introduction of a thin interlayer of amorphous $\mathrm{Al}_{2} \mathrm{O}_{3}$ within the stack suppresses the leakage below the common DRAM criteria of $100 \mathrm{nA} / \mathrm{cm}^{2}$ and leads to a reliability improvement, but lowers the effective dielectric constant and therefore, the capacitance equivalent thickness (CET). Replacing $\mathrm{Al}_{2} \mathrm{O}_{3}$ with a material with a higher k-value will enhance the stack CET without degrading the leakage performance. Therefore, it is important to find a material with a crystallization temperature high enough to remain amorphous 
after electrode deposition. One promising candidate would be strontium oxide. SrO has a reported dielectric constant of 16, a crystallization temperature higher than $600{ }^{\circ} \mathrm{C}$ [7], and a band gap of $\sim 4.5 \mathrm{eV}$ mixed in amorphous $\mathrm{HfO}_{2}$ [8]. Table 1 summarizes the band gap, k-value and crystallization temperature of crystal $\mathrm{ZrO}_{2}$ and amorphous $\mathrm{Al}_{2} \mathrm{O}_{3}$ and $\mathrm{SrO}$.

This work presents results of pure $\mathrm{ZrO}_{2}$ film as well as metal-insulator-metal (MIM) stacks with $\mathrm{Al}_{2} \mathrm{O}_{3}$ (ZAZ) and $\mathrm{SrO}(\mathrm{ZSrZ})$ as interlayer.

TABLE I Dielectric material properties

\begin{tabular}{cccc}
\hline \hline & Gap (eV) & $\boldsymbol{\varepsilon}_{\mathbf{0}}$ & Cryst. Temp $\left({ }^{\circ} \mathbf{C}\right)$. \\
\hline $\mathbf{Z r O}_{2}$ & $5.8[10]$ & $\sim 40$ (crystalline) & $\sim 400$ \\
$\mathbf{a - A l _ { 2 } \mathbf { O } _ { 3 }}$ & $6-6.2[8]$ & $\sim 25$ (amorphous) & $>850$ \\
$\mathbf{a - S r O}$ & $\sim 4.5[8]$ & 9 & $>600$ \\
\hline \hline
\end{tabular}

\section{EXPERIMENTAL PROCEDURE}

The whole MIM stack was prepared by an in-situ physical vapor deposition (PVD) process in a Bestec UHV cluster tool. First, TiN was deposited at a substrate temperature of $300{ }^{\circ} \mathrm{C}$ as bottom electrode (BE). Afterwards, the $\mathrm{ZrO}_{2} / \mathrm{X} / \mathrm{ZrO}_{2}$ stack was prepared at room temperature. Finally, the TiN top electrode (TE) was grown again at $300{ }^{\circ} \mathrm{C}$. After MIM stack deposition crystallization of the $\mathrm{ZrO}_{2}$ was induced by a $450{ }^{\circ} \mathrm{C}$ anneal for 10 minutes in a nitrogen atmosphere. Annealing at this temperature is required to reach fully crystallized $\mathrm{ZrO}_{2}$ layers with a k-value of $\sim 40$. To form the individual capacitor structures, platinum dots were evaporated onto the top TiN layer in a Bestec evaporation chamber. The size of the pads was determined by the Pt dots used as a hard mask for the structuring of the TE. Thus, the top TiN layer was removed outside the pads with a diluted standard clean 1 (SC-1) solution. The oxide thickness of the investigated samples was 5- $10 \mathrm{~nm}$.

Electrical characterization was done using a semiautomatic probe station from Cascade Microtech and an Agilent B1500A Semiconductor Device Parameter Analyzer, equipped with Source Monitor Units and a Capacitance Measurement Unit. To characterize the material reliability conventional voltage ramp stress VBD tests were performed, with an additional sense current measurement at a fixed low voltage. 


\section{RESULTS AND DISCUSSION}

Fig. 1 shows the current-voltage (I-V) characteristic for the three investigated material stacks as black lines. Measurements were performed at elevated temperature of $125^{\circ} \mathrm{C}$ to activate trap related phenomena like stress induced leakage current (SILC). The voltage was ramped from $0 \mathrm{~V}$ up to dielectric breakdown, while the voltage step size was $100 \mathrm{mV}$. It can be seen that for both interlayers the leakage current is reduced compared to the pure $\mathrm{ZrO}_{2}$ film. For positive bias the stress current is slightly higher, which can be related to a TiOx based interface formation at the BE during TE deposition and anneal [11]. This fact and the presence of SILC for positive bias at the TE will be discussed later in this section.

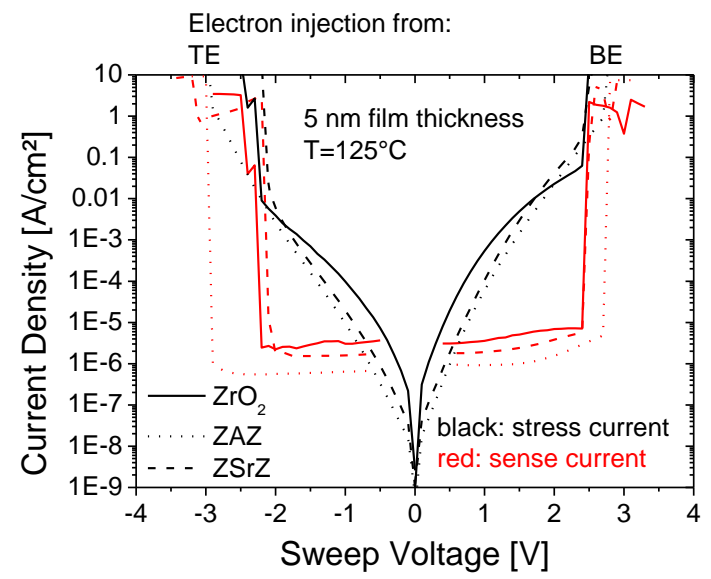

FIG. 1. Voltage-current traces of $5 \mathrm{~nm} \mathrm{ZrO}_{2}, \mathrm{ZAZ}$ and $\mathrm{ZSrZ}$ films at a temperature of $125^{\circ} \mathrm{C}$. At certain current level a fixed SILC sense voltage is applied.

As shown in Fig. 1, the leakage could be reduced to a comparable level for both $\mathrm{Al}_{2} \mathrm{O}_{3}$ and $\mathrm{SrO}$ interlayer. Fig. 2 presents the leakage over CET for all three film stacks. Leakage and CET were measured at $25{ }^{\circ} \mathrm{C}$. The physical film thickness was in the range of 5 to $10 \mathrm{~nm}$.

For comparison reason, data from literature for atomic layer deposited (ALD) films annealed at a comparable thermal budget are included [12]. ALD is the favored deposition technique for DRAM manufacturing, because it allows fabrication of 3-dimensional, high aspect ratio capacitor structures with a conformal and homogeneous thickness. However, here PVD was used to enable straightforward implementation of different sputter materials in the material stack without time consuming process development as expected for ALD deposition. Nevertheless, as presented in Fig. 2, the CET values of the PVD layers are close to results of ALD films. 


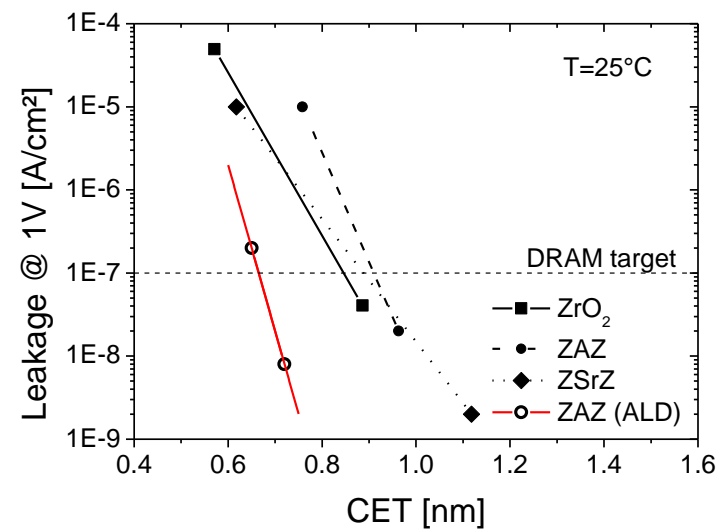

FIG. 1. CET-Leakage plot for $\mathrm{ZrO}_{2}, \mathrm{ZAZ}$ and $\mathrm{ZSrZ}$ films measured at $25^{\circ} \mathrm{C}$. Physical film thickness was in the range of 5 to $10 \mathrm{~nm}$.

For the PVD film stack it can be seen, that at DRAM target leakage of $100 \mathrm{nA} / \mathrm{cm}^{2}$ the CET of the ZAZ films increases compared to pure $\mathrm{ZrO}_{2}$. For the stack with the $\mathrm{SrO}$ interlayer the CET could be improved, due to the higher k-value of SrO. DRAM capacitors need even small improvements to be able to scale to the next technology node. These results are indicating the enhancement potential which might be further increased by going to ALD-based dielectric layers.

To get a closer look on the breakdown and SILC behavior for the investigated film stacks, the voltage ramp was extended by a sensing step. At a certain current level a fixed sense voltage was applied after each stress step for SILC monitoring. As presented in Fig. 1(red), the sense current shows a bias dependent behavior. For negative bias at the TE, meaning electron injection from the TE, the sense current is almost stable up to hard breakdown. For BE injection, the sense current traces show a SILC related continuous increase before breakdown.

As mentioned before, the metal/dielectric interface is different for top and bottom electrode. Therefore, the tunneling barrier height is expected to be different and also a higher interface roughness can lead to large electric field peaks at the bottom metal/dielectric interface [13]. To investigate the influence of the tunneling barrier height, internal photoemission was measured on the pure TiN/ZrO $2 / \mathrm{TiN}^{\mathrm{M}} \mathrm{MIM}$ stack [14]. Fig. 3 shows the results for positive bias at the TE. First, the electron IPE shows a threshold of $\sim 2 \mathrm{eV}$, which corresponds to the energy barrier between the Fermi level of the metal and the bottom of the $\mathrm{ZrO}_{2}$ conduction band. Two additional features can be seen at $\sim 4 \mathrm{eV}$ and $\sim 5.8 \mathrm{eV}$. The value of $5.8 \mathrm{eV}$ represents the band gap of pure $\mathrm{ZrO}_{2}$, whereas, as published by Lucovsky et al., the $4 \mathrm{eV}$ value hints on the formation of a TiOx network at the interface to the TiN electrode [15]. 


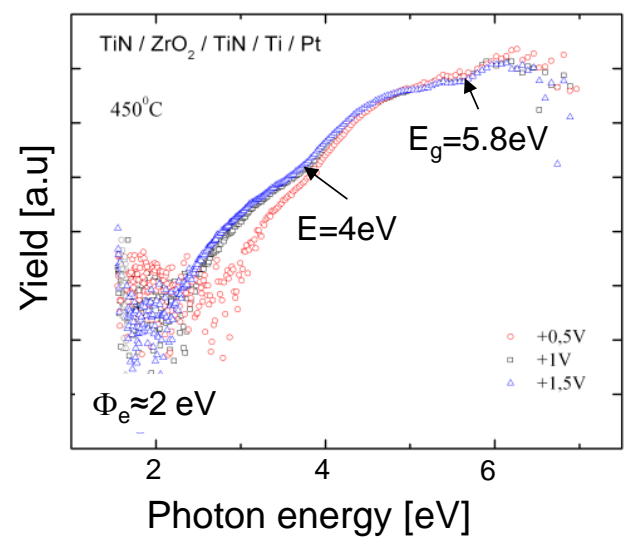

FIG. 3. Photocurrent quantum yield as a function of photon energy measured on a TiN/ZrO2/TiN MIM capacitor with positive bias applied to the top metal electrode.

Fig. 4 presents the Weibull distribution of the breakdown voltage (VBD) for the $5 \mathrm{~nm}$ film thickness measured at $125^{\circ} \mathrm{C}$. The pure $\mathrm{ZrO}_{2}$ film shows small VBD's and wide distribution, especially for negative stress bias. The VBD of the $\mathrm{ZAZ}$ increases compared to the $\mathrm{ZrO}_{2}$ film. In addition, the slope of the distribution is improved. For the ZSrZ film VBD increases as well, showing even higher values when negative stress bias is applied. Nonetheless, it could be shown that an introduction of $\mathrm{SrO}$ interlayer leads to comparable results as the incorporation of an $\mathrm{Al}_{2} \mathrm{O}_{3}$ interlayer. As visible in table 2, the reliability of the dielectric stack was improved compared to $\mathrm{ZrO}_{2}$.for a $\mathrm{ZSrZ}$ dielectric and at the same time the $\mathrm{Sr}$ interlayer caused a CET-leakage behavior similar to the best values of pure $\mathrm{ZrO}_{2}$.

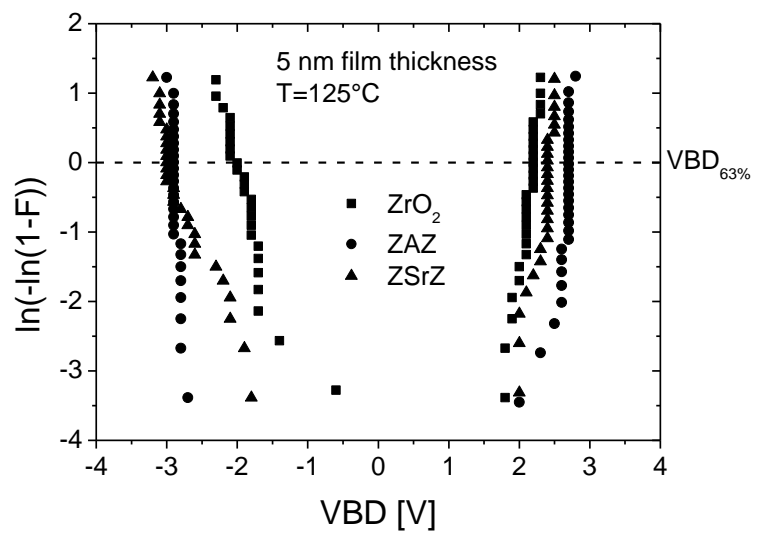

FIG. 4. Weibull distribution of the breakdown voltage of $5 \mathrm{~nm} \mathrm{ZrO}, \mathrm{ZAZ}$ and $\mathrm{ZSrZ}$ films at a temperature of $125^{\circ} \mathrm{C}$. 
TABLE II VBD and leakage of different zircon dioxide based dielectrics

\begin{tabular}{cccc}
\hline \hline & $\mathbf{V B D}_{\mathbf{6 3} \%}(+\mathrm{V})[\mathbf{V}]$ & $\mathbf{V B D}_{\mathbf{6 3} \%}(-\mathrm{V})[\mathrm{V}]$ & $\begin{array}{c}\text { Leakage current } \\
\text { density @ 1V [A/cm²] }\end{array}$ \\
\hline $\mathbf{Z r O}_{2}$ & $2.2 \mathrm{~V}$ & $-2 \mathrm{~V}$ & $8 \mathrm{E}-5$ \\
$\mathbf{Z A Z}$ & $2.7 \mathrm{~V}$ & $-2.9 \mathrm{~V}$ & $1 \mathrm{E}-5$ \\
$\mathbf{Z S r Z}$ & $2.4 \mathrm{~V}$ & $-3 \mathrm{~V}$ & $1 \mathrm{E}-5$ \\
\hline
\end{tabular}

\section{CONCLUSION}

The thickness scaling of DRAM capacitor dielectrics is close to its physical limits at the $20 \mathrm{~nm}$ technology node. Therefore, the requirements of the DRAM capacitor dielectric are quite challenging for future generation nodes. $\mathrm{A} \mathrm{ZrO}_{2}$ based material is the material of choice due to his high-k value of $\sim 40$, in crystalline phase, and its band gap of $5.8 \mathrm{eV}$. Previously, an $\mathrm{Al}_{2} \mathrm{O}_{3}$ interlayer was introduced to improve leakage and reliability, but with the disadvantaged of a lowering of the effective k-value. Here, film properties were further enhanced by including SrO to increase the overall k-value of the capacitor dielectric, which resulted in an improved CETleakage ratio compared to ZAZ film stacks. Additionally, a promising influence on VBD was shown. Summarizing, the replacement of $\mathrm{Al}_{2} \mathrm{O}_{3}$ in actual DRAM dielectric stacks by another material with a higher kvalue, is an interesting pathway for further stack improvement to enable future DRAM generation nodes. Further dielectric and electrode materials need to be screened to reach future requirements, but every incremental step is important to fine-tune the electrical properties.

\section{REFERENCES}

${ }^{1}$ D. -S. Kil, H. -S. Song, K. -J. Lee, K. Hong, J. -H. Kim, K. -S. Park, S. -J. Yeom, J. -S. Roh, N. -J. Kwak, H. C. Sohn, J. -W. Kim, and S. -W. Park, "Development of New TiN/Z $\mathrm{ZrO}_{2} / \mathrm{Al}_{2} \mathrm{O}_{3} / \mathrm{ZrO}_{2} / \mathrm{TiN}$ Capacitors Extendable to $45 \mathrm{~nm}$ Generation DRAMs Replacing $\mathrm{HfO}_{2}$ Based Dielectrics,” Dig. Tech. Pap. - Symp. VLSI Technol. 2006, pp.38-39

${ }^{2}$ J. -Y. Seo, J. -E. Seok, H. -J. Kim, H. -J. Kim, H. -S. Park, J. -E. Jeon, and W. -S. Lee, "Reliability acceleration model of stacked $\mathrm{ZrO}_{2}-\mathrm{Al}_{2} \mathrm{O}_{3}-\mathrm{ZrO}_{2}$ MIM capacitor with cylinder type," Proc. MIEL 2008, 2008, pp.521-524.

${ }^{3}$ H. J. Cho, Y. D. Kim, D. S. Park, E. Lee, C. H. Park, J. S. Jang, K. B. Lee, H. W. Kim, Y. J. Ki, I. K. Han, and Y. W. Song, "New TIT capacitor with $\mathrm{ZrO}_{2} / \mathrm{Al}_{2} \mathrm{O}_{3} / \mathrm{ZrO}_{2}$ dielectrics for $60 \mathrm{~nm}$ and below DRAMs," Solid-State Electron. 51, 2007, pp.1529-1533

${ }^{4}$ M. Popovici, J. Swerts, A. Redolfi, B. Kaczer, M. Aoulaiche, I. Radu, S. Clima, J.-L. Everaert, S. Van Elshocht, M. Jurczak, "Low leakage Ru-strontium titanate-Ru metal-insulator-metal capacitors for sub-20 nm technology node in dynamic random access memory," APL 104, 2014, p. 082908

${ }^{4}$ S. Kupke, S. Knebel, U. Schroeder, 3 S. Schmelzer, U. Böttger, T. Mikolajick, „Reliability of $\mathrm{SrRuO}_{3} / \mathrm{SrTiO}_{3} / \mathrm{SrRuO}_{3}$ Stacks for DRAM Applications," IEEE EDL vol. 33, no.12, 2012, pp.1699-1701, Dec. 2012

${ }^{6} \mathrm{X}$. Zhao and D. Vanderbilt, "First-principles study of structural, vibrational, and lattice dielectric properties of hafnium oxide," Phys. Rev. B 65, 2002, pp.233106

${ }^{7}$ M. Galtier, A. Montaneret G. Vidal, "Phonons optiques de $\mathrm{CaO}, \mathrm{SrO}, \mathrm{BaO}$ au centrede la zone de brillouin a 300 et 17K," J. Phys. Chem. Solids 33, 1972, pp.2295-2302 
${ }^{8}$ S. Shamuilia, V. V. Afanas'ev, A. Stesmans, I. McCarthy, S. A. Campbell, M. Boutchich, M. Roeckerath, T. Heeg, J. M. J. Lopes, J. Schubert, "Photoconductivity of Hf-based binary metal oxide systems," JAP 104, 2008, p. 114103

${ }^{9}$ J.L. Jacobson and E.R. Nixon, "Infrared dielectric response and lattice vibrations of Calcium and Strontium Oxides“, J.Phys.Chem.Solids 29, 1968, p. 967

${ }^{10}$ J. Robertsen,"Band offsets of wide-band-gap oxides and implications for future electronic devices," J. Vac. Sci. Technol. B 18, 2000, pp.1785-1791

${ }^{11}$ W. Weinreich, R. Reiche, M. Lemberger, G. Jegert, J. Müller, L. Wilde, S. Teichert, J. Heitmann, E. Erben, L. Oberbeck, U. Schröder, A.J. Bauer, H. Ryssel, "Impact of interface variations on J-V and C-V polarity asymmetry of MIM capacitors with amorphous and crystalline $\mathrm{Zr}_{(1-\mathrm{x})} \mathrm{Al}_{\mathrm{x}} \mathrm{O}_{2}$ films," Microelectron. Eng. 86, 2009, pp.1826-1829.

${ }^{12}$ K.-R. Yoon, K.-V. Im, J.-H. Yeo, E.-A. Chung, Y.-S. Kim, C.-Y. Yoo, S.-T. Kim, U-I. Chung, J.-T. Moon, "Performance and reliability of MIM (Metal-Insulator-Metal) Capacitors with $\mathrm{ZrO}_{2}$ for 50nm DRAM Application," SSDM, 2005, pp.188-189

${ }^{13}$ N. Gaillard, L. Pinzelli, M. Gros-Jean, A. Bsiesy, "In situ electric field simulation in metal/insulator/metal capacitors", Appl. Phys. Lett. 89, 2006, p. 133506

${ }^{14} \mathrm{~V}$. V. Afanas'ev, A. Stesmans, "Internal photoemission at interfaces of high-k insulators with semiconductors and metals," Jour. Appl. Ph. 102, 2007, p. 081301

${ }^{15}$ G. Lucovsky, C. C. Fulton, Y. Zhang, Y. Zou,J. Luning, L. F. Edge,J. L. Whitten, R. J. Nemanich, H. Ade, D. G. Schlom, V. V. Afanas'ev, A. Stesmans, S. Zollner, D. Triyoso, B. R. Rogers, "Conduction band-edge States associated with the removal of d-state degeneracies by the Jahn-Teller effect," IEEE TDMR, vol.5, no.1, 2005, pp. 65,83 